\title{
Desempeño en Funciones Ejecutivas de Adultos Mayores: Relación Con su Autonomía y Calidad de Vida
}

\author{
Executive Functions Performance In Old Adults: \\ Relationship With Autonomy And Quality Of Life
}

\author{
Dra. Nancy Lepe-Martínez, Lic. Francisca Cancino-Durán,2 Lic. Fernanda Tapia-Valdés, Lic. Pamela Zambrano-Flores, \\ Lic. Patricia Muñoz-Veloso, Dra. Ivonne Gonzalez-San Martín, ${ }^{3}$ Dr. Carlos Ramos-Galarza ${ }^{4}$
}

\begin{abstract}
Resumen
A lo largo del ciclo vital, el ser humano es capaz de controlar de manera consciente sus pensamientos, emociones y conducta de manera que se ajusten a las demandas del entorno, lo que se va perdiendo con la vejez, afectando la vida independiente y autónoma de las personas, impactando negativamente su calidad de vida. El propósito de esta revisión es determinar las funciones ejecutivas que se ven más deterioradas con el paso de los años. Se usó el método de revisión narrativa para recopilar los artículos que abordaban la relación entre funciones ejecutivas y calidad de vida en adultos mayores sin daño cognitivo grave. Los resultados muestran que las funciones ejecutivas que más se afectan con los años son la atención, memoria operativa y fluidez verbal, implicadas en la búsqueda y actualización de información; flexibilidad cognitiva, responsable de generar modificaciones en la conducta, pensamiento y razonamiento, fundamentales en el funcionamiento cognitivo eficiente. Además, se reporta un enlentecimiento en el razonamiento, déficit inhibitorio, déficit de trasmisión y sensorio-perceptivo. Se concluye que las funciones ejecutivas son sensibles al proceso de envejecimiento y afectan progresivamente la autonomía y calidad de vida de los adultos mayores. Se cree relevante generar programa de mantenimiento de estas funciones cognitivas, como una formar de promover un envejecimiento exitoso.
\end{abstract}

Palabras claves: Funciones ejecutivas, adulto mayor, calidad de vida, autonomía en adultos mayores.

\section{Abstract}

Throughout the life cycle, the human being is able to consciously control his thoughts, emotions and behavior in a way that adjusts to the demands of the environment, which is lost with old age, affecting the independent and autonomous life of people, negatively impacting their quality of life. The purpose of this review is to determine the executive functions that are most impaired over the years. The narrative review method was used to collect articles that addressed the relationship between executive functions and quality of life in older adults without severe cognitive impairment. The results show that the executive functions that are most affected over the years are attention, working memory and verbal fluency, involved in the search and updating of information; cognitive flexibility, responsible for generating modifications in behavior, thinking and reasoning, fundamental in efficient cognitive functioning. In addition, a slowdown in reasoning, inhibitory deficit, transmission deficit and sensory-perceptive is reported. It is concluded that executive functions are sensitive to the aging process and progressively affect the autonomy and quality of life of older adults. It is considered relevant to generate a maintenance program for these cognitive functions, as a way to promote successful aging.

Keywords: Executive functions, older adults, quality of life, autonomy in older adults.

Rev. Ecuat. Neurol. Vol. 29, N 1, 2020

'Académica Facultad de Ciencias de la Educación, Universidad Católica del Maule. Talca, Chile.

${ }^{2}$ Carrera de Educación Especial, Universidad Católica del Maule, Talca, Chile.

${ }^{3}$ Centro Interdisciplinario de Innovación Educativa (CIED)

Universidad Santo Tomás. Chile

${ }^{4}$ Académico Facultad de Psicología, Pontificia Universidad Católica del Ecuador. Quito, Ecuador.
Correspondencia:

Dra. Nancy Lepe-Martinez

Avenida San Miguel 3605, Talca, Chile.

E-mail:nlepe@ucm.cl 


\section{Introducción}

Las Funciones Ejecutivas (FE) comprenden un conjunto de habilidades cognoscitivas cuyo principal objetivo es facilitar la adaptación en las distintas etapas de la vida a situaciones nuevas y complejas, más allá de las conductas consideradas habituales y automáticas. Éstas comprenden estrategias cognitivas, tales como la solución de problemas, formación de conceptos, planeación y memoria de trabajo. Específicamente las FE se relacionan con destrezas para establecer metas, flexibilidad del pensamiento, inhibición de respuestas automáticas para ejecutar otra más ajustada al contexto, como también con la capacidad de desarrollar planes de acción, la autorregulación del comportamiento y la fluidez verbal.2 Se pueden entender como las rutinas responsables de la monitorización y regulación de los procesos cognitivos durante la realización de tareas cognitivas complejas.

Las definiciones de FE son muy variadas y pocas veces se encuentran coincidencias entre estas. Sin embargo, existen criterios supra ordinales que permiten comprender mejor el cómo abordarlas, es decir, desde la perspectiva ontológica (lo que las FE son), ${ }_{4}^{4}$ pragmática (lo que hacen) ${ }^{5}$ y nominales (aludiendo a que constructo se trata). ${ }^{6}$ A este escenario se suma la complejidad de ser un concepto que en el contexto de la literatura neuropsicológica aborda el estudio de fenómenos cercanos a la psicología funcionalista, hacen necesario tener presente los criterios que la definen y como esto influye en la revisión.

Desde lo ontológico las FE se han definido como los "procesos que asocian ideas, movimientos y acciones simples y los orientan a la resolución de conductas complejas." ${ }^{4}$ Del mismo modo Lezak, las define como las "capacidades mentales esenciales para llevar a cabo una conducta eficaz, creativa y aceptada socialmente" (p. 673). Estas abarcan en la práctica una serie de procesos cognitivos entre los que destacan la anticipación, elección de objetivos, planificación, selección de la conducta, autorregulación, autocontrol y uso de la retroalimentación o más bien conocido como feedback?

Desde el punto de vista funcional se puede afirmar que en el córtex prefrontal se encuentran las funciones cognitivas más complejas y evolucionadas del ser humano, a las que se les atribuye un papel esencial en actividades tan importantes como la creatividad, la ejecución de actividades complejas, la toma de decisiones y el juicio ético y moral. ${ }^{7}$ La FE se encuentran se encuentran en estas áreas cerebrales prefrontales involucradas en las estrategias cognitivas, tales como la solución de problemas, formación de conceptos, planeación y memoria de trabajo.

Por otro lado, las FE mantienen una relación jerárquica con capacidades cognitivas como atención y memoria, ejerciendo un control de su funcionamiento para adecuarlo a las metas conductuales de las personas. Lezak $^{9}$ fue la primera en sistematizar el concepto de dife- renciándolo de las capacidades cognitivas básicas como la memoria, la atención y el lenguaje. Para Lezak ${ }^{10}$ las FE se preguntan por el cómo de una conducta, a diferencia de las capacidades básicas que se preguntan por el qué de la conducta. Se trata de un proceso que comienza con la identificación de sus necesidades por parte de una persona, le sigue la planificación de la conducta para satisfacerlas y luego la ejecución de esta.

En relación con los procesos que integran las $\mathrm{FE}$, se pueden dividir en procesos fríos y calientes. Los procesos fríos se relacionan más con respuestas que implican habilidades cognitivas desprovistas de contexto, los procesos calientes se vinculan a respuestas afectivas a situaciones que son significativa, es decir la capacidad de regulación de las emociones. ${ }^{11}$

Según Lezak ${ }^{12}$ la conducta humana está estructurada por tres grandes sistemas que interactúan estrechamente entre sí, el sistema cognitivo que facilita la adquisición y el procesamiento de la información por medio de diferentes dominios cognitivos; como la atención, memoria, lenguaje, percepción y pensamiento. Mientras que el sistema emocional está relacionado con las emociones, sentimientos y motivaciones que le dan un matiz especial tanto a la cognición como a la conducta. Por último, el sistema de funciones ejecutivas se encarga de funciones de coordinación como la planeación, la organización y la verificación de acciones y de estrategias que facilitan la expresión de la conducta ajustada socialmente.

\section{Funciones Ejecutivas}

\section{Memoria de trabajo}

La memoria implica un conjunto de habilidades que permiten registrar, almacenar y recuperar la información cuando la situación lo demanda, es una función cognoscitiva muy compleja y tiene un papel relevante para el adecuado funcionamiento de otros procesos cognoscitivos. Estas habilidades de la memoria dependen de diferentes sistemas cerebrales descritos en diversos modelos clasificatorios: tiempo, modalidad de la información y conciencia entre otros. En relación con el tiempo, puede ser clasificada en memoria de corto y largo plazo, con relación a la modalidad de información, la memoria se clasifica en verbal o visual, y en relación con la consciencia en memoria explícita o implícita. Del mismo modo, los dos grandes sistemas que clasifican a la memoria son la memoria no declarativa y declarativa. ${ }^{13}$

La memoria que interviene en la realización de importantes tareas cognitivas tales como la comprensión del lenguaje, lectura y el pensamiento es la memoria de trabajo que se define como un sistema que mantiene y manipula temporalmente la información. ${ }^{14}$ Figueroa, Lagos y García ${ }^{15}$ afirman que la memoria de trabajo tiene una capacidad de almacenamiento limitada, que posibilita la retención, seguimiento y manipulación de la infor- 
mación, empleando para ello estrategias de codificación, almacenamiento, mantenimiento, control, inhibición y digitalización de la información.

Las etapas de la memoria son la codificación, almacenamiento y recuerdo o evocación. ${ }^{13}$ En la codificación se realiza el registro de la información en el cerebro, la atención y concentración permite incorporar la información en la memoria a corto plazo, desde ahí la información puede ser trasladada al almacén de largo plazo, las principales estrategias usadas para el registro de la información son la repetición y asociación de significados. En la etapa de almacenamiento a la información se le asigna un significado en la estructura del conocimiento, lo que facilita la recuperación de la información cuando sea necesario, tanto la codificación como el almacenamiento son fundamentales para el proceso de aprendizaje. El proceso de recuperación permite traer a un estado presente la información guardada en la memoria de corto o largo plazo, lo que permite usar la información para resolver una tarea específica.

La memoria de trabajo forma parte de los subsistemas de memoria no declarativa, caracterizada por ser consciente y estar conformada por sistemas de la cognición, destacando que es un almacén temporal y de procesamiento activo de la información, siendo su función no almacenar datos, sino manipularlos. Baddeley ${ }^{16}$ definió la memoria de trabajo como un almacén temporal que permite la manipulación de la información para llevar a cabo un amplio rango de actividades cognitivas complejas. Su capacidad de almacenamiento es limitada, ya que la información se desvanece y desaparece en segundos al concluir la tarea.

La estructura de la memoria de trabajo. Se estructura en cuatro subsistemas: el primero relacionado encargado del mantenimiento y manipulación de la información auditivo verbal (bucle fonológico); el segundo de naturaleza espacial, encargado del mantenimiento y manipulación de la información visual y espacial, (agenda visoespacial); el tercero encargado de entregar los recursos atencionales a los diferentes subsistemas (ejecutivo central); por último, retén episódico, que ha sido incorporado recientemente, que se encarga del almacenamiento simultáneo de información de los dos primeros componentes y de la memoria a largo plazo de modo que se crea una representación multimodal y temporal de la situación actual, de modo que se crea una representación multimodal y temporal de la situación actual. ${ }^{13}$

\section{Fluidez verbal}

La fluidez verbal en su sentido más amplio consiste en la capacidad de producir un lenguaje espontáneamente fluido, sin excesivas pausas que reflejen dificultades de acceso al léxico. El correcto funcionamiento de esta habilidad requiere de la participación de las capacidades de almacenamiento semántico, de recuperación de la información y la indemnidad de las FE. ${ }^{17}$ En síntesis, la fluidez, es la velocidad y precisión en la búsqueda y actualización de la información, así como la producción de elementos específicos en un tiempo eficiente; es un importante atributo de la corteza prefrontal y se relaciona con la FE de productividad. ${ }^{18}$ Además de cumplir una función compleja que implica diferentes procesos cognitivos para la producción lingüística, compromete la capacidad de producción verbal controlada y programada, estrategias de búsqueda, organización y eliminación de respuestas previamente dadas, ${ }^{19}$ también utiliza procesos cognitivos como la atención, la memoria de trabajo, la flexibilidad, la velocidad de procesamiento de la información, la iniciativa y el monitoreo de producción, el funcionamiento ejecutivo y la activación del lóbulo frontal ${ }^{20}$

Dentro de los tipos de fluidez verbal se encuentran la fluidez verbal semántica y la fluidez verbal fonológica. La fluidez verbal-semántica presenta incrementos continuos al menos hasta los 14-15 años de edad. La fluidez verbal depende más que otras FE de las influencias lingüísticas, psicolingüísticas y cognitivas siendo más sensible a las variables de escolaridad y demanda educativa. ${ }^{21}$

\section{Flexibilidad cognitiva}

La flexibilidad cognitiva es considerada por Diamond $^{22}$ como "el proceso ejecutivo responsable de generar modificaciones en las conductas y pensamientos en contextos dinámicos de las personas, sujetos a rápidos cambios y fluctuaciones, dependiendo de las demandas del entorno" (p. 23). Por ello, la posibilidad de cambiar eficiente y velozmente cuando las circunstancias lo demandan constituye un rasgo esencial del comportamiento adaptativo y ajustado a los objetivos. Sin embargo, la posibilidad de adaptarnos a los cambios del ambiente no sólo requiere de flexibilidad cognitiva, sino también de otros procesos de control ejecutivos como la memoria de trabajo y la inhibición.

Uno de los métodos más utilizados para evaluar la flexibilidad cognitiva es el paradigma de cambio de tarea, procedimiento que se caracteriza por una disminución en el desempeño de los ensayos que implica un cambio en relación con los ensayos donde la tarea se repite, siendo la inhibición el principal mecanismo explicativo de esta materia. Es en esta línea que una de las propuestas teóricas más difundidas es la Teoría de la Inercia de la Tarea, que consistente en la presencia del coste del cambio, deriva de la activación persistente de los procesos establecidos durante la ejecución de los ensayos de la tarea anterior. Por lo tanto, propone que el coste de cambio depende más de la tarea desde la que se cambia, que de la tarea hacia la que se cambia. ${ }^{23}$

Por último, la flexibilidad cognitiva es considerada un componente ejecutivo que resulta imprescindible para la adaptación del individuo al medio y por ende para el logro de las distintas metas conductuales. Es por ello, el 
análisis de cualquier factor o proceso que puede afectar su desarrollo o funcionamiento constituye un tema de interés para la investigación actual. En este sentido, la función inhibitoria ocupa un rol protagónico para todas las actividades que debe desarrollar el ser humano en relación con las demandas establecidas por su entorno dependiendo de las tareas establecidas a desarrollar. ${ }^{23}$

\section{Control inhibitorio}

Existe cierto consenso en considerar a la inhibición como un constructo multidimensional o no unitario. Es por esto, que en la literatura se distingue entre distintos procesos inhibitorios con características funcionales y operativas discriminadas ${ }^{24-25}$ En este sentido, la taxonomía más difundida, es el Modelo Inhibitorio Tripartito ${ }^{22}$ que distingue tres procesos inhibitorios: inhibición comportamental, inhibición cognitiva e inhibición perceptual. La principal función de la inhibición comportamental es la supresión de respuestas prepotentes. Mientras este proceso contribuye al control del comportamiento los otros dos procesos inhibitorios -la inhibición cognitiva y perceptual- se aplican a la cognición, pues intervienen de manera activa regulando la activación o inhibición de representaciones y pensamientos.

La inhibición cognitiva, es la responsable de disminuir el nivel de activación de las representaciones mentales prepotentes, de los pensamientos de carácter intrusivo o, lo que es lo mismo de la información irrelevante de la memoria de trabajo ${ }^{22}$ En líneas generales, este término se refiere a un proceso de control que interviene reduciendo la accesibilidad de aquellas representaciones de la memoria que resultan irrelevantes y que generan interferencia sobre las que se consideran relevantes para el logro de las metas actuales. ${ }^{26}$ Por último, la inhibición perceptual es el mecanismo que permite focalizar la atención en los estímulos, atenuando la interferencia vinculada a la presencia de otros estímulos presentes en el ambiente. Este proceso inhibitorio cumple un rol fundamental en la atención selectiva funcionando incluso para algunos autores como términos equivalentes debido a su rol principal en la atenuación del efecto de interferencia de distractores externos en un contexto de competencia estimular. ${ }^{22,27}$

\section{Funciones ejecutivas a lo largo del ciclo vital}

Las FE son las funciones que tardan más tiempo en desarrollarse, estas están presentes durante todo el ciclo de la vida. Se dice que, durante los primeros años de vida, el niño parece vivir en un tiempo presente con reacciones solamente a estímulos que se encuentran en su alrededor inmediato. Posteriormente, es capaz de representar estímulos del pasado, planear el futuro, y analizar un problema desde distintos parámetros, buscando soluciones apropiadas a cada uno de ellos, ${ }^{28}$ esta capacidad para planear y solucionar problemas establece el inicio de las
FE. Se ha encontrado que es hacia los 5 meses de edad cuando el bebé sabe que un juguete continúa existiendo aun cuando está fuera de su vista. Sin embargo, es a partir de los 9 meses de edad aproximadamente que buscará de manera activa el juguete ${ }^{29}$

En pruebas de acción-inhibición se encontró que la capacidad para inhibir respuestas es significativamente es mejor en niños de 9 y 12 años, comparados con niños de 6 a 8 años. La superioridad del control atencional en niños de 9 a 11 años, fue corroborada por Brocki y Bohlin. ${ }^{30} \mathrm{La}$ incapacidad para inhibir respuestas automáticas, en niños pequeños no parece ser el resultado de una falta de comprensión de las reglas de una tarea particular, sino más bien a su falta de control de respuestas automáticas. Livesey y Morgan $^{31}$ estudiaron el desempeño de niños de 4 y 5 años en pruebas de acción-inhibición y encontraron que a pesar de que los participantes eran capaces de realizar una discriminación verbal entre las instancias de acción e inhibición y que entendían las reglas de la actividad, sin embargo, no eran capaces de realizar la tarea correctamente al no poder inhibir la respuesta motora inapropiada.

La planeación es la capacidad para identificar y organizar una secuencia de eventos con el fin de lograr una meta especifica. ${ }^{32}$ Desde los 3 años el niño entiende que es capaz de formular propósitos verbales simples relacionados con eventos familiares, siendo también apto para poder solucionar problemas y desarrollar estrategias para prevenir problemas futuros. ${ }^{33}$ Este tipo de planeación se complejiza más a la edad de 7 y 11 años, quienes desarrollan un plan de acción mucho más organizado y eficaz.4

La flexibilidad cognoscitiva se define como la habilidad para cambiar rápidamente de una respuesta a otra empleando estrategias alternativas. Implica normalmente un análisis de las consecuencias de la propia conducta y un aprendizaje de sus errores. ${ }^{35}$ Se estima que la flexibilidad cognoscitiva aparece entre los 3 y los 5 años cuando al niño se le facilita cambiar de una regla a otra, por ejemplo, en tareas de clasificación de objetos ${ }^{36}$ Algunos autores creen que, hasta los 7 años el niño continúa presentando dificultades en pruebas de clasificación en las que se debe mantener acceso mental a varias reglas para poder hacer cambios de una regla a otra durante el desempeño de la tarea.$^{37}$ De Luca y colaboradores ${ }^{38}$ encontraron que la habilidad para cambiar de una estrategia a otra alcanza el nivel del adulto hacia entre los 8 y los 10 años.

En cuanto a la fluidez verbal, se puede dividir en fluidez verbal fonológica (o alfabética) y semántica. La primera exige la producción de palabras que se inician con un fonema o letra y la segunda requiere que las palabras generadas pertenezcan a una categoría semántica (ejemplo: animales). Algunos autores sugieren que la fluidez verbal se mantiene sin cambio por la edad gracias a que depende de una fuente de conocimiento verbal que se mantiene intacta con el paso de los años. ${ }^{39}$ Siendo poco sensible al paso del 
tiempo y solamente se hacen evidentes en la octava década de la vida. ${ }^{40}$ Las habilidades de fluidez verbal semántica y fonológica parecen alcanzar su máximo desarrollo entre la adolescencia y la adultez temprana.

\section{Concepto de Adulto Mayor Qué es ser adulto mayor}

La Organización Mundial de la Salud ${ }^{41}$ postula que el concepto de adulto mayor está dado por el envejecimiento natural de las personas a partir de los 60 años. Se espera que para el año 2020, el número de personas de esta edad o más, será superior al de niños menores de 5 años. En este sentido, es un desafío garantizar sistemas sanitarios y sociales que respondan a las características de los cambios demográficos a nivel mundial.

En Chile, el Ministerio de Salud y Protección Social, ${ }^{42}$ define a las personas adultas mayores como aquellos sujetos de derecho, socialmente activos, con garantías y responsabilidades respecto de sí mismas, su familia y su sociedad, son su entorno inmediato y con las futuras generaciones. Respecto al proceso de envejecimiento humano individual, Alonso, Sansó, Díaz-Canel, Carrasco y Oliva, ${ }^{43}$ mencionan que éste se entiende producto de la suma de dos tipos de envejecimiento: el primario, intrínseco o per se y el secundario. El envejecimiento primario es el proceso o grupo de procesos responsables del conjunto de cambios observados con la edad en los individuos de una especie y no relacionados con la presencia de enfermedad. Se centra en los mecanismos genéticos, moleculares y celulares que intervienen en el proceso de envejecimiento y que, de expresarse adecuadamente, condicionan lo que se ha denominado "envejecimiento con éxito."

El envejecimiento secundario, hace referencia al que se produce en los seres vivos cuando son sometidos a la acción de fenómenos aleatorios y selectivos, que ocurren a lo largo del tiempo de vida y que interaccionan con los mecanismos y cambios propios del envejecimiento primario para producir el "envejecimiento habitual." Los principales indicadores de este envejecimiento secundario son los problemas de salud de carácter crónico y los cambios adaptativos para mantener la homeostasis del medio interno. La investigación en esta área abarca tanto las causas, prevención, desarrollo, manifestación, pronóstico y tratamiento de la enfermedad y de sus consecuencias, como lo relacionado con hábitos y estilos saludables de vida.

Sea cual sea el tipo de envejecimiento considerado, la característica fundamental común a cualquiera de ellos es la pérdida de la reserva funcional, que condiciona una mayor susceptibilidad a la agresión externa, al disminuir los mecanismos de respuesta y su eficacia para conservar el equilibrio del medio interno. Esta disminución de la respuesta se manifiesta inicialmente sólo bajo circunstancias de intenso estrés para luego pasar a manifestarse ante mínimas agresiones. ${ }^{43}$
Según se pierde la reserva funcional, la susceptibilidad es mayor, aumentando la posibilidad de que cualquier cosa desencadene en una pérdida de función, discapacidad y dependencia. En general, las políticas internacionales van orientadas a un cambio en la concepción de la vejez, considerando a ésta como una etapa de vida activa, en la que se puede lograr el máximo de autonomía individual y la posibilidad de la autorrealización. ${ }^{44}$

El proceso de envejecimiento del organismo humano no debe reducirse a un mero proceso biológico, se debe analizar en el contexto de la naturaleza compleja del hombre que es un ser biológico/cultural/social/mental y espiritual integradamente..$^{45}$ A esto se debe agregar que existe una gran variación entre individuos, pues cada persona envejece de modo diferente por las características físicas y fisiológicas, por su estructura de personalidad e historia de vida, y por el contexto socioeconómico en el que se desarrolla. ${ }^{46}$ El envejecimiento está comúnmente relacionado con el deterioro cognitivo. Por ello, es necesario investigar los elementos que pueden contribuir a las intervenciones preventivas en la rehabilitación cognitiva, con el objetivo de preservar la calidad de vida de las personas de edad avanzada. ${ }^{47}$

\section{Funciones Ejecutivas en Adultos Mayores}

En el envejecimiento se producen cambios cognitivos, psicológicos y afectivos. Esta etapa de la vida se asocia generalmente a una disminución en la capacidad de la memoria y aprendizaje; un incremento en la tasa de olvido y una disminución en la capacidad para adquirir nueva información. Uno de los aspectos más sobresalientes en la memoria de los ancianos es la dificultad para recordar hechos recientes, a pesar de describir con relativa facilidad eventos antiguos, particularmente aquellos relacionados con situaciones emocionales. ${ }^{48,49,50}$

Como consecuencia de este proceso de envejecimiento, se producen cambios en el funcionamiento cognitivo, precisamente en aquellas funciones más complejas, cambios que se manifiestan en un enlentecimiento del procesamiento de la información, disminución en la capacidad atencional, declives en aspectos de la memoria y en las denominadas $\mathrm{FE}^{48,49,50}$

Las FE se consideran fundamentales en esta etapa de la vida, ya que permiten que la persona sea autónoma, tome diversas decisiones, planifique y programe sus actividades diarias. Las $\mathrm{FE}$ son habilidades cognoscitivas sensibles al proceso de envejecimiento, ya que los procesos cognitivos mediados por el lóbulo prefrontal sufren un deterioro con la edad, lo que no sucede con habilidades mediadas por áreas cerebrales más posteriores. ${ }^{51,52}$

Es importante recalcar que aquellos adultos mayores que están constantemente activos cognitivamente, es decir, tienen alguna actividad laboral, mantienen un interés por aprender actividades nuevas o practican algún deporte, presentan mejores rendimientos respecto a aquellos que 
no lo hacen o no consideran estas actividades en su vida. Investigaciones demuestran que las FE son las más sensibles al proceso de envejecimiento $5^{3}$ De hecho, se observa una vulnerabilidad especial del lóbulo prefrontal a los efectos de la edad, por lo que se afirma que los procesos mediados por el lóbulo frontal son los primeros en sufrir deterioro con la edad avanzada. ${ }^{54}$

Las FE y el aprendizaje, muestran cambios con el paso de los años. De hecho, los resultados de las pruebas neuropsicológicas que evalúan el funcionamiento ejecutivo de muchos adultos mayores sanos presentan, en general, indicios de disfunción, incluyendo excesiva rigidez mental, alteración de la atención, enlentecimiento del procesamiento de la información y dificultades para la toma de decisiones, modificaciones que inevitablemente inciden para que el proceso de aprendizaje se realice de manera diferente a etapas anteriores de la vida. ${ }^{53}$

En síntesis, las FE son parte de las funciones cognitivas más complejas y exteriorizan cambios en el envejecimiento. Las actividades de estas funciones cumplen un papel fundamental en el funcionamiento cognitivo, ya que contribuyen a que la persona aprenda, tome decisiones, se proyecte, planifique, se maneje autónomamente, entre otras habilidades importantes para el ejercicio cotidiano. ${ }^{55}$

\section{Funciones ejecutivas descendidas en adultos mayores}

Con el paso del tiempo, las actividades diarias a realizar van disminuyendo en cantidad y esfuerzos, lo que hace que el cerebro se encuentre menos estimulado y activo, lo que limita su funcionamiento. Una de las habilidades en deterioro más frecuentes es la pérdida de capacidad para inhibir el procesamiento automático de estímulos familiares incompatibles con la tarea en curso, como sucede en las situaciones que requieren atender sólo a una parte de la información concurrente, debiendo ser ignorada la información distractora competitiva. ${ }^{56,57,58,59}$

También se evidencia en esta etapa de la vida deterioro de la capacidad para coordinar la ejecución simultánea de dos tareas, ${ }^{60,61}$ alteración relacionada con la incapacidad para inhibir el procesamiento automático de estímulos, dado que ambas implican descontrol de la atención, en un caso para concentrarla de forma selectiva y en otra para distribuirla de manera coordinada al enfrentarse a dos fuentes de información. A esto se le debe agregar todos los estímulos externos presentes al momento de la ejecución de las tareas, lo que pone una mayor dificultad para poder hacerlo de manera eficiente.

Estudios han detectado pérdida en la capacidad de razonamiento, tanto inductivo como deductivo, ${ }^{62,59}$ en la memoria operativa o de trabajo, ${ }^{63,64,65}$ en la fluidez verbal ${ }^{66}$ y de plasticidad o flexibilidad cognitiva, ${ }^{67,68}$ entre otras disfunciones ejecutivas.

Algunas de las habilidades y/o destrezas que se encuentran en proceso de descenso en adultos mayores son la velocidad de procesamiento, donde se produce un enlentecimiento en el procesamiento de la información cuando los adultos mayores son sometidos a diversas tareas, lo que los hace emitir respuestas de manera más lenta. Un enlentecimiento evidente es el procesamiento sintáctico, donde el tiempo utilizado en el proceso lector de oraciones con diferente estructura sintáctica aumenta respecto de la misma lectura en personas jóvenes ${ }^{69}$ (Pp. 78).

Otra habilidad que empieza a presentar descenso en esta etapa de la vida es el control inhibitorio, el envejecimiento debilita los procesos inhibitorios que regulan la atención sobre los contenidos de la memoria operativa. Ello afecta a una amplia gama de actuaciones cognitivas entre las cuales se incluyen la comprensión y producción del lenguaje. , $^{71}$ La memoria, no solo debe activar y procesar ciertas unidades de información, sino también debe filtrar los contenidos no pertinentes para que no se produzca interferencia o confusión. Para ello, deben operar simultáneamente dos funciones: una función de acceso, que restringe el ingreso de información irrelevante a la memoria operativa y una función de supresión, que elimina la información que ha dejado de ser útil o que estuvo en estado de latencia y que finalmente no se utiliza. Si estos mecanismos inhibitorios de la atención se debilitan, puede suceder que mientras se está procesando la información surjan pensamientos intrusivos o se hagan asociaciones no relevantes, elementos que, al competir por recursos de procesamiento, reducen la capacidad funcional de la memoria de trabajo afectando la comprensión y el recuerdo. ${ }^{72}$

Se produce, además, disminución de la capacidad de la memoria operativa, entendida como un sistema de memoria de recursos limitados, capaz de almacenar, manipular y evocar información, la que experimenta una disminución en su capacidad de trabajo, hecho que impone limitaciones a la habilidad de las personas mayores para comprender o producir oraciones semántica o sintácticamente complejas. ${ }^{73,74,75}$ Se requieren recursos de memoria sea para planificar el contenido o generar las ideas, mantener activas las representaciones gramaticales, fonológicas u ortográficas durante la codificación y mantener el control ejecutivo de todo el proceso.

Estudios en esta materia, muestran que sujetos mayores obtienen tasas más bajas de desempeño que los jóvenes en tareas que requieren comprensión o recuerdo inmediato de oraciones de estructura sintáctica compleja. Se evidencia, además una tendencia general de los mayores a producir oraciones gramaticalmente más simples, cuando escriben o participan en conversaciones o son entrevistados. Estos resultados han sido interpretados como indicadores de esa declinación. Esos estudios han medido la capacidad de la memoria operativa usando básicamente la prueba de amplitud de memoria de Daneman y Carpenter. ${ }^{76}$

En los adultos mayores se produce también déficit en la trasmisión. En este sentido, la teoría del déficit de 
transmisión ${ }^{77}$ se basa en un modelo conexionista localista que concibe el lenguaje como una vasta red de conexiones entre unidades organizadas en un subsistema fonológico y un subsistema semántico, donde muchos de los cambios cognitivos asociados al envejecimiento podrían deberse al debilitamiento de las conexiones entre las representaciones en la memoria. Así lo sugieren los datos sobre procesamiento semántico existentes, los que muestran que los mayores tienen representaciones semánticas tan ricas como los jóvenes ${ }^{7}$ Diferente es la situación en el sistema fonológico, donde las conexiones entre las unidades son singulares y por ello más vulnerables a los déficits de transmisión. Un buen ejemplo es el fenómeno conocido como en la punta de la lengua, algo frecuente en adultos mayores, que puede ser explicado por déficit en la recuperación de unidades fonológicas de la palabra que se quiere decir. $^{79}$

Finalmente, es posible encontrar también en adultos mayores un descenso sensorio-perceptivo. La teoría del déficit sensorio-perceptivo o de la señal degradada ${ }^{80}$ plantea que la declinación de los procesos sensoriales y perceptivos asociada al envejecimiento, principalmente relacionados con la audición y visión, producen entradas erróneas o incompletas de información, lo que afecta la codificación fonológica y ortográfica. Esto se debe a fallas en la selección léxica, lo que afecta la compresión y producción del lenguaje.

Tabla 1. Resumen características evidenciables de FE descendidas en adultos mayores.

\begin{tabular}{|ll|}
\hline Habilidades en descenso & Características \\
\hline Enlentecimiento & $\begin{array}{l}\text { Dificultad en la velocidad de } \\
\text { procesamiento al momento de una } \\
\text { tarea. }\end{array}$ \\
\hline Déficit inhibitorio & $\begin{array}{l}\text { Dificultad en los procesos de } \\
\text { atención, afectando la comprensión } \\
\text { y producción del lenguaje. } \\
\text { Dificultad en la reducción de la } \\
\text { capacidad funcional de la memoria } \\
\text { de trabajo afectando la compren- } \\
\text { sión o el recuerdo. }\end{array}$ \\
\hline $\begin{array}{l}\text { Disminución de la } \\
\text { capacidad de la memoria }\end{array}$ & $\begin{array}{l}\text { Disminución en la capacidad de } \\
\text { trabajo de la memoria. } \\
\text { operativa }\end{array}$ \\
$\begin{array}{l}\text { Requiere recursos de memoria } \\
\text { para planificar el contenido y } \\
\text { generar ideas. }\end{array}$ \\
\hline Déficit de transmisión & $\begin{array}{l}\text { Debilitamiento de las conexiones } \\
\text { entre las representaciones en la } \\
\text { memoria. }\end{array}$ \\
\hline Déficit sensorio-perceptivo & $\begin{array}{l}\text { Declinación de procesos } \\
\text { sensoriales y perceptios asociada } \\
\text { principalmente, a la audición y } \\
\text { visión. } \\
\text { Produce falla en el proceso de } \\
\text { selección léxica y en procesos } \\
\text { subsiguientes sumados a una } \\
\text { declinación en los procesos de } \\
\text { percepción que influyen en la } \\
\text { comprensión y producción del } \\
\text { lenguaje. }\end{array}$ \\
\hline
\end{tabular}

Fuente: Elaboración propia.

\section{Funciones Ejecutivas y Calidad de Vida en Adultos Mayores}

Las FE se ven afectadas con el envejecimiento, trayendo consigo una disminución en la flexibilidad mental, menor precisión y lentitud al cambiar de actividad, reducción del razonamiento práctico ante tareas complejas y disminución en la capacidad de solución de problemas lógicos por la presencia de desorganización y redundancia en el pensamiento. ${ }^{81}$ Así mismo, resultados de pruebas neuropsicológicas que evalúan el funcionamiento ejecutivo de adultos mayores sanos muestran que se encuentran indicios de disfunción, incluyendo excesiva rigidez mental, alteración de la atención, enlentecimiento del procesamiento de la información y dificultades para la toma de decisiones, modificaciones que inevitablemente inciden en el proceso de aprendizaje, lo obliga a que el aprendizaje se realice de manera diferente a etapas anteriores de la vida. ${ }^{53}$

El deterioro en la FE afecta de manera directa a la calidad de vida del adulto mayor, definido según Ardilla ${ }^{82}$ como "un estado de satisfacción general, derivado de la realización de las potencialidades de la persona" considerando aspectos como lo son el bienestar físico, psicológico y social. La dimensión social y psicológica del adulto mayor, puede verse afectada por la connotación negativa de la vejez, que genera exclusión por parte de la sociedad. De esta manera, se dice que el adulto mayor pierde el rol que desempeñaba dentro de su círculo, por lo que se tiende a acoplar a grupos sociales, los que son más fragmentados debido a las condiciones de la edad. Así pues, a nivel psicológico se desarrolla una crisis del desarrollo acompañada por una serie de preocupaciones que exigen un alto nivel de adaptación por parte del adulto mayor ${ }^{83}$

En el ámbito social, aquellos adultos que continúan realizando una actividad laboral, mantenido el interés por aprender, demuestran tener un mejor rendimiento en la prueba de fluidez verbal que aquellos que no realizan ningún tipo de actividad, lo que implicaría que los primeros almacenarían un nivel más elevado de conceptualización y abstracción del pensamiento que los segundos, por ende, tendrían mayor capacidad para encontrar una estrategia eficiente de resolución de una tarea o un problema que les permita la adaptación a la nueva situación84; por lo que la participación social y las relaciones interpersonales genera un efecto positivo en el individuo.

Manes y Torralva ${ }^{85}$ miembros del Instituto de Neurología Cognitiva (INECO), han manifestado por medio de sus investigaciones que el adulto mayor puede mantenerse activo cognitivamente, siendo capaz de seguir aprendiendo, gracias a la neuroplasticidad. Sumado a ello, las estrategias de aprendizajes ayudan a procesar la información de manera significativa, generando en los adultos mayores sentimientos de autoeficacia ${ }^{86}$ 
En una investigación sobre correlación entre el control inhibitorio y la memoria, ${ }^{87}$ en una muestra de 73 adultos mayores con una edad media de 72,44 años. Los resultados indican que la memoria a Corto Plazo (MCP) fue similar en los cuatro grupos; y en cuanto a la Memoria a Largo Plazo (MLP) las medias de los adultos mayores de 86 a 93 años fueron mayores. En la memoria sensorial (MS) las medias fueron semejantes, siendo menores las del grupo de 69 a 76 años. Sin embargo, en la memoria operativa (MO) las medias de los grupos 2 y 3 (69-76 y 77-85) resultaron significativamente bajas con respecto a los otros dos grupos. También llama la atención que los mejores resultados fueron para los adultos mayores de 86 a 93 años. Con respecto al Control Inhibitorio (CI) los dos grupos intermedios también mostraron los resultados más bajos. En esta variable resultó realmente interesante que las medias del grupo de adultos mayores de 86 a 93 años, contrario a lo que pudiera esperarse, fueron significativamente mayores que las de los grupos restantes.

Los resultados mostraron correlaciones directas entre control inhibitorio y memoria sensorial tanto verbal como visuo-espacial. Además, existió una correlación positiva con la memoria operativa visuo-espacial altamente significativa; sin embargo, no se encontraron correlaciones significativas con la memoria operativa verbal. Se halló una correlación negativa entre el control inhibitorio y el número de errores en el reconocimiento de palabras cuando existe relación semántica, lo que puede explicar la relación entre control inhibitorio y memoria de trabajo verbal, pues al existir un mejor control inhibitorio (expresado en una mayor puntuación en $\mathrm{PC}$ ) será menor el número de errores relacionados semánticamente, lo que implica un mejor funcionamiento de la memoria de trabajo verbal. ${ }^{87}$

González, Rubio y Grasso ${ }^{88}$ utilizaron pruebas de memoria donde se daba estimulación y se les exponía aun ambiente enriquecedor, las cuales fueron aplicadas a 70 adultos mayores cuyas edades comprendían desde los 60 hasta los 85 años, de ambos sexos, encontrando que al realizar una evaluación dinámica (con el uso de la mediación) los adultos mayores pueden mejorar su rendimiento cognitivo y beneficiarse de las intervenciones y ayudas de un experto evaluador. En este estudio se observó que las intervenciones utilizadas fueron altamente significativas y lograron diferenciar a los sujetos que las recibieron de aquellos que no las recibieron. Ello indicaría que, ante condiciones de estimulación óptima, los adultos mayores evidencian un rendimiento cognitivo mayor.

En lo que respecta la calidad de vida de los adultos mayores, Melguizo, Acosta y Castellano, ${ }^{89}$ aplicaron una encuesta de tipo descriptiva a 514 adultos mayores de los cuales se obtuvo los siguientes resultados; los hombres reportaron un mejor bienestar físico y emocional, mien- tras que las mujeres reportaron un mejor autocuidado y plenitud espiritual. Se menciona, además, que, si se favorece y se capacita a los adultos mayores a generar sus propios ingresos, se podría esperar una mejor percepción acerca de su calidad de vida.

Aguilar, de los Ángeles y Herrera, ${ }^{90}$ en un estudio aplicado a 48 personas adultos mayores, con edades comprendidas entre los 60 y 89 años. Los resultados revelaron que los componentes de la FE más comprometidos fueron la memoria de trabajo y la planeación, mientras los más conservados, la flexibilidad mental y fluidez verbal tanto en su expresión fonológica como semántica. Si bien el presente estudio no tuvo en cuenta un análisis distinguiendo grupos etarios, fue posible observar disminuida la capacidad para prever o anticipar el resultado de la respuesta a fin de solucionar la tarea, concebir cambios a partir de las circunstancias presentes, analizar alternativas, sopesar y hacer elecciones.

Ball, et al. ${ }^{11}$ publicaron en Norteamérica un estudio en donde se evaluaron si 3 intervenciones de entrenamiento cognitivo en memoria, razonamiento inductivo y velocidad del procesamiento de información mejoran la capacidad mental y el funcionamiento diario de 2.832 adultos mayores independientes entre los 65 y 94 años, quienes residen en viviendas para ancianos y centros comunitarios de seis áreas metropolitanas de los Estados Unidos. Los resultados apoyan la eficacia y la durabilidad de las intervenciones de entrenamiento cognitivo en la mejora de las habilidades cognitivas específicas.

Montejo $^{92}$ estudió los resultados de un programa de entrenamiento en memoria con el método de la Unidad de Memoria del Ayuntamiento de Madrid (UMAM) en una población de 1.083 sujetos mayores de 65 años con alteraciones de memoria, a los cuales les realizó una evaluación pre-entrenamiento, otra post y una más a los seis meses. Se concluyó que el método multifactorial de entrenamiento de memoria UMAM produjo una mejoría objetiva y subjetiva de la memoria en mayores con alteraciones de ésta, manteniéndose la mejoría a los 6 meses.

Valencia, López, Tirado, Zea, Lopera y Rupprecht93 en la ciudad de Medellín, realizaron un estudio con el objetivo de evaluar los efectos cognitivos de un entrenamiento combinado de memoria y psicomotricidad denominado "Independencia en la Vejez", en una muestra de 95 adultos mayores sanos (49 en el grupo de intervención y 46 en el grupo control), antes y después del programa de entrenamiento de 20 sesiones. Los resultados mostraron efectos positivos del entrenamiento, específicamente en la atención selectiva y la velocidad de procesamiento de información.

Un estudio realizado por Garamendi, Delgado y Amaya ${ }^{94}$ quienes desarrollaron un programa de entrenamiento con el propósito de demostrar que éste mejora el 
deterioro cognitivo en 68 sujetos, con una edad igual o mayor de 60 años de ambos sexos, que presentan deterioro en la Prueba de Mini-Examen Cognoscitivo de Lobo (MEC) y/o en la Evaluación Neuropsicológica Breve en español (NEUROPSI). A los participantes se les aplicó un programa de ejercicios prácticos de estimulación cognitiva durante 20 sesiones de 60 minutos por sesión. Los resultados evidencian que los adultos mayores mostraron un porcentaje de mejoría en la prueba de MEC de 15.2\% y en la prueba Neuropsi un $16.33 \%$. Así pues, se pudo concluir que el deterioro cognitivo mejoró con la aplicación del programa de ejercicios prácticos de estimulación cognitiva en adultos mayores.

\section{Comentarios Finales}

El estudio de las FE ha demostrado que éstas se desarrollan de manera progresiva desde el nacimiento hasta la adultez. En cuanto a la capacidad para cambiar de una estrategia a la otra se alcanzan alrededor de los 10 años. En este sentido se puede decir que las FE son un conjunto de habilidades cognitivas que guían los pensamientos, emociones y acciones, lo que permita la realización de respuestas adecuadas - exitosas y/o eficientes - a una tarea o conjunto de tareas. Son funciones que se desarrollan desde la primera infancia y tienen su mejor desempeño desde la adolescencia.

Esta investigación narrativa tuvo como objetivo determinar las FE que se van deteriorando en adultos mayores. En este sentido, se puede indicar que la etapa de la vida de adulto mayor implica variados desafíos y desajustes, tanto en aspectos emocionales, psicológicos, como sociales. Las personas presentan un declive en las funciones cognitivas. Las FE más deficientes en adultos mayores, son atención, memoria operativa y fluidez verbal, implicadas en la búsqueda y actualización de información; flexibilidad cognitiva responsable de generar modificaciones en la conducta, pensamiento y razonamiento, fundamentales en el funcionamiento cognitivo. Además, se reporta un enlentecimiento en el razonamiento, déficit inhibitorio, déficit de trasmisión y sensorio-perceptivo, todo lo cual afecta la velocidad y calidad de las respuestas que los adultos mayores dan a las tareas del entorno.

Se cree relevante seguir indagando en las FE más descendidas en los adultos y los efectos que estas producen en la conducta de las personas, con la finalidad de diseñar planes de estimulación que potencien las FE deterioradas y permitan así mejorar la autonomía y calidad de vida de los adultos mayores, haciendo de esta etapa de la vida un momento para seguir activos física y mentalmente, sintiéndose competentes para disfrutar de su entorno social y familiar.

\section{Referencias}

1. Ardila, A., \& Surloff, C. Dysexcutive syndromes. San Diego: Medlink: Neurology, 2007.

2. Rosselli, M., Jurado, M., y Matute, E. Las funciones ejecutivas a través de la vida. Revista Neuropsicología, Neuropsiquiatría y Neurociencias, 2008, 8 (1), 23-46.

3. Miyake, A., Friedman, N., Emerson, M., Witzki, A., Howerter, A. \& Wager, T. The unity and diversity of executive functions and their contributions to complex frontal lobe tasks: A latent variable analysis. Cognitive Psychology,2000, 41, 49-100.

4. Shalice, T. Specific impairments of planing. Philosophical transcripts of the royal society of London, 1982, 298, 199-290.

5. Sholberg, M., \& Mateer, C. Remediation of executive functions impairments. Introduction to cognitive rehsbilitation, 1989, 17, 232-263.

6. Lezak, M. Relationship between personality disorders, social disturbances and physical disability following traumatic brain injury. $\mathrm{J}$ head trauma rehabilitation, 1987, 2 (1), 257-280.

7. Pelegrín, C., \& Tirapu, J. Neuropsiquiatría del daño prefrontal traumático. Monografías de Psiquiatría, 1995, 7, 11-21.

8. Price, B., Daffner, K., Stowe, R., \& Mesulam, M. The comportamental learning disabilities of earlylobe damage. Brain, 1990, 113, 1383-1393.

9. Lezak, M. Europsychological assessment. (2nd ed.) New York: Oxford University Press, 1983.

10. Lezak, M. Europsychological assessment. (1st ed). New York: Oxford University Press, 1995.

11. Zelazo, P., Qu, L. \& Muller, U. Hot and cool aspects of executive function: relations in early development. En W. Schneider, R. Schumann y B. Sodian (Eds.), Young children's cognitive development: Interrelationships among executive Junctioning, working memory, verbal ability, and theory of mind. Mahwah: Lawrence Eribaum Associates Publishers, 2004.

12. Lezak, M. D. Executivefunction and motor performance. In M. D. Lezak, D. B. Howieson, \& D. W. Loring (Eds.), Neuropsychological assessment (4th ed., pp. 611-646). New York: Oxford University Press, 2004.

13. Aguilar, S., Gutiérrez, L., \& Samudio, M. Estimulación de la atención y la memoria en adultos mayores con deterioro cognitivo. México: Permanyer, 2018.

14. Tirapu, J., Muñoz, J., \& Pelegrín, C. Funciones ejecutivas: de una integración conceptual. Revista Neurol, 2002, 34 (7), 673-685.

15. Figueroa, C., Lagos, R., \& García, M. Nivel educativo, edad y funcionamiento ejecutivo. Areté, 2017, 17, 1-8.

16. Baddeley, A. Is Working Memory Still Working? (P. Frensch, Ed.) European Psychologist, 2002, 7 (2), 85-97. 
17. Butman, J., Allegri, R., Harris, P., \& Drake, M. Fluencia verbal en español. Datos normativos en Argentina, 2000, 60, 561-4.

18. Lezak, M., Howieson, D., \& Loring, D. Neuropsychological assessment (4a. ed.). New York: Oxford University Press, 2004.

19. Benjumea, M., Ocampo, E., Vega, J., Hernández, J., \& Tamayo, D. Fluidez verbal en estudiantes del grado de $11^{\circ}$ de las instituciones educativas Alejandro Vélez Barrientos y José Manuel Restrepo del Municipio de Envigado, según la prueba neuropsicología de las funciones ejecutivas BANFE. Katharsis, 2008, 22, 63-85.

20. Narbona, J. \& Muller, C. El lenguaje del niño. Desarrollo normal, evaluación y trastornos. España, Barcelona: Masson S.A., 1997.

21. Flores, J., Castillo, R., \& Jiménez, N. Desarrollo de funciones ejecutivas, de la niñez a la juventud. Anales de psicología, 2014, 30 (2), 463-473.

22. Diamond, A. Executive Functions. Annual Review of Psychology. 2013, 64, 135-168.

23. Introzzi, I., Canet, L., Montes, S., López, S., \& Mascarello, G. Procesos inhibitorios y flexibilidad cognitiva: evidencia a favor de la Teoría de la Inercia Atencional. INT. J. PSYCHOL, 2015, 8 (2),60-74.

24. Friedman, N. P., \& Miyake, A. The relations among inhibition and interference control functions: A latent-variable analysis. Journal of Experimental Psychology, 2004, 133, 101-135.

25. Nigg, J. T. On inhibition/disinhibition in developmental psychopathology: Views from cognitive and personality psychology and a working inhibition taxonomy. Psychological Bulletin, 2000, 126, 220-246.

26. Anderson, M., \& Bjork, R. Mechanisms of inhibition in long-term memory: A new taxonomy. In D. Dagenbach \& T. Carr (Eds.), Inhibitory processes in attention, memory, and language. New York: Academic Press, 1994.

27. Eriksen, B. A., \& Eriksen, C. W. Effects of noise letters upon the identification of a target letter in a nonsearch task. Perception \& Psychophysics, 1974, 16, 143-149.

28. Zelazo, P., Craik, F., \& Booth, L. Executive functions across the lifespan. Acta Psychologica, 2004, 115, 167-183.

29. Baillargeon, R., Spelke, E., \& Wasserman, S. Object permanence in five- month-old infants. Cognition, 1985, 20, 191- 208.

30. Brocki, K., \& Bohlin, G. Executive functions in children aged 6 to 13: A dimensional and developmental study. Developmental Neuropsychology, 2004, 26(2), 571-593.

31. Livesey, D., \& Morgan, G. The development of response inhibition in 4- and 5-year-old children. Australial Journal of Psychology, 1991, 43, 133-137.
32. Lezak, M., Howieson, D., Loring, D., \& Fischer, J. Neuropsychological assessment. USA: Oxford University Press, 2004.

33. Hudson, J., Shapiro, L., \& Sosa, B. Planning in the real World: Preeschool children's scripts and plans for familiar events. Child Development, 1995, 66, 984-998.

34. Levin, H., Culhane, K., Hartmann, H., Evankovich, K., Mattson, A., \& Harwood, H. Developmental changes in performance on tests of purported frontal lobe functions. Developmental Neuropsychology, 1991, 7, 377-396.

35. Anderson, V., Levin, H., \& Jacobs, R. Executive functions after frontal lobe injury: A developmental perspective. En D. T. Stuss, \& R.T. Knight (Eds.), Principles of frontal lobe function. New York: Oxford University Press, 2002.

36. Epsy, K. The shape school: Assessing executive function in preschool children: Developmental Neuropsychology, 1997, 13, 495-499.

37. Anderson, V., Northam, E., Hendy, J., \& Wrenall, J. Developmental Neuropsychology: A clinical approach. New York: Psychology Press, 2001.

38. De Luca, C. R., Wood, S. J., Anderson, V.,Buchanan, J., Proffitt, T. M., Mahony, K., \& Pantelis, C. Normative data from the Cantab: Development of executive function over the lifespan. Journal of Clinical and Experimental Neuropsychology, 2003, 25, 242-254.

39. Crawford, J., Bryan, J., Luszcz, M., Obonsawin, M., \& Stewart, L. The executive decline hypothesis of cognitive aging: Do executive deficits qualify as differential deficit and do they mediate agerelated memory decline. Aging, Neuropsychology, and Cognition, 2000, 7, 9-31.

40. Emery, O. Language and aging. Experimental Aging Research, 1985, 11, 3-60.

41. Organización Mundial de la Salud. Envejecimiento y salud. Recuperado el 23 de marzo de 2020 de: https:// www.who.int/es/news-room/fact-sheets/detail/envejecimiento-y-salud.

42. Ministerio de Salud y Protección Social. Envejecimiento y vejez. Recuperado el 23 de marzo de 2020 de: https:/www.minsalud.gov.co/proteccionsocial/ promocion-social/Paginas/envejecimiento-vejez.aspx.

43. Alonso Galbán, P., Sansó Soberats, F. J., Díaz-Canel Navarro, A. M., Carrasco García, M., \& Oliva, T. Envejecimiento poblacional y fragilidad en el adulto mayor. Revista cubana de salud pública, 2007, 33(1), 1-17.

44. Belenger, M.V. y Aliaga, F. Autonomía funcional y ocupación del tiempo libre en personas mayores. Rev. Rol de Enfermería, 2000, 23(3), 231-234.

45. Aponte, V. Calidad de vida en la tercera edad. Universidad Católica Boliviana "San Pablo": La Paz, 2015.

46. Cornachione, M. Psicología evolutiva de la vejez. Córdoba: Talleres gráficos de Germán Marcelo Ferrero, 1999. 
47. Nascimento, F., Argimon, I., \& Lopes, M. Atualidades do idoso no mercado de trabalho. Psicologia. com.pt., 2006, 1-7.

48. Craik, F. I., \& Salthouse, T. A. The Handbook of Aging and Cognition: Third Edition (3 ed.). (F. I. Craik, \& T. A. Salthouse, Edits.) New York: Psichology Press, 2011.

49. Donoso, A., Behrens, M., \& Venegas, P. Deterioro cognitivo leve: seguimiento de 10 casos. Chilena de neuro-psiquiatría, 2003, 41 (2), 117-122.

50. Neugarten, B. Psychological aspects of aging and illness. (T. Stern, Ed.) Psychosomatic, 1984, 25 (2), 123-125.

51. Daigneault, S., Braun, C., \& Whitaker, H. Early effects of normal aging on perseverative and non-perseverative prefrontal measures. Developmental Neuropsychology, 1992, 8 (1), 99-114.

52. West, R. An application of prefrontal cortex function theory to cognitive aging. Psychological Bulletin, 1996, 120 (2), 272-292.

53. Jurado, M., Matute, E., \& Rosselli, M. (2008). Las funciones ejecutivas a través de la vida. Revista Neuropsicología, Neuropsiquiatría y Neurociencias, 2008, 8 (1), 23-46.

54. Dempster, F. N. The rise and fall of the inhibitory mechanism: Toward a unified theory of cognitive development in aging. Developmental Review, 1992, 12, 45-75.

55. Burin, D., Drake, M. y Harris, P. Evaluación neuropsicológica en adultos. Bs. As: Paidós SAICF, 2007.

56. Levinoff, E.J., Saumier, D. \& Cherkowe, H. Focused attention deficits in patients with Alzheimer's disease and mild cognitive impairment. Brain and Cognition, 2005, 57, 127- 130.

57. Spieler, D., Balota, D. \& Faust, M. Stroop performance in health younger and older adults and in individuals with dementia of the Alzheimer's type. Journal of Experimental Psychology: Human Perception and Performance, 1996, 22, 461-479.

58. Amieva, H., Phillips, L., Della Sala, S. \& Henry, J. Inhibitory functioning in Alzheimer's disease. Brain, 2004, 127, 949-964.

59. Waltz, J., Knowlton, B., Back-Madruga, C., McPherson, S., Masterman, D. \& Miller, B. Relational integration and executive function in Alzheimer's disease. Neuropsychology, 2004, 18, 296-303.

60. Della Sala, S. \& Logie, R. H. Theoretical and practical implications of dual task performance in Alzheimer's disease. Brain, 2001, 124, 1479-1481.

61. Logie, R. H., Cocchini, G., Della Sala, S. \& Baddeley, A. D. Is there a specific executive capacity for dual task coordination? Evidence from Alzheimer's disease. Neuropsychology, 2004, 18, 504-513.

62. Helmes, E. \& Ostbye, T. Beyond memory impairment: Cognitive changes in Alzheimer's disease. Archives of Clinical Neuropsychology, 2002, 17, 179-193.
63. Collette, F. \& Van der Linden, M. Mémoire de travail et maladie d'Alzheimer. En M. Ergis, M. C. Gély-Nargeot y M. Van der Linden (Eds.), Les troubles de la mémoire dans la maladie d'Alzheimer. Marseille: Solal, 2005.

64. Germano, C. \& Kinsella, G. J. Working memory and learning in early Alzheimer's disease. Neuropsychology Review, 2005, 15, 1-10.

65. Morris, J. C. The Clinical Dementia Rating (CDR): current version and scoring rules. Neurology, 1993, 43, 2412-2414.

66. Stokholm, J., Vogel, A., Gade, A. \& Waldemar, G. Heterogeneity in executive impairment in patients with very mild Alzheimer's disease. Dementia and Geriatric Cognitive Disorders, 2006, 22, 54-59.

67. Fernández-Ballesteros, R., Zamarrón, M., Tárraga, L., Moya, R., \& Iñiguez, J. Cognitive plasticity in healthy, mild cognitive impairment (MCI) subjects and Alzheimer's disease patients: A research project in Spain. European Psychologist, 2003, 8, 148-159.

68. Perry, R. J., Watson, P. \& Hodges, J. R. The nature and staging of attention dysfunction in early (minimal and mild) Alzheimer's disease: relationship to episodic and semantic memory impairment. Neuropsychologia, 2000, 38, 252-271.

69. Riffo, B. \& Véliz M. Informe final proyecto Fondecyt 1050591. Comisión Nacional de Ciencia y Tecnología (Conicyt), Chile, 2008.

70. Hasher, L., \& Zacks, R. Working memory, comprehension and aging: A review and a new view. En G. H. Bower (Ed.), The psychology of learning and motivation (pp.193-225). New York: Academic Press, 1988.

71. Zacks, R. \& Hasher, L. "Cognitive gerontology and attentional inhibition: A reply to Burke and McDowd". En Journal of Gerontology: Psychological Sciences, 1997, 52B, 274-283.

72. Zacks, R., Hasher, L. \& Li, K. Z. H. "Human memory". En F.I.M. Craik \& T A. Salthouse (eds.), Handbook of aging and cognition. Hillside, N.J.: L. Erlbaum, 2000.

73. Carpenter, P. A., Miyaki, A. \& Just, M.A. "Working memory constraints in comprehension: Evidence from individual differences, aphasia and aging". En M. Gernsbacher (ed.), Handbook of Psycholinguistics. San Diego, C. A.: Academic Press, 1994, 1075-1122.

74. Just, M. A., \& Carpenter, P. A. A capacity theory of comprehension: individual differences in working memory. Psychological review, 1992, 99(1), 122.

75. Kemtes, K. A., \& Kemper, S. Aging and resolution of quantifier scope effects. The Journals of Gerontology Series B: Psychological Sciences and Social Sciences, 1999, 54(6), 350-360.

76. Daneman, M. \& Carpenter, P.A. Individual Differences in working memory and reading. Journal of Verbal Learning and Verbal Behavior, 1980, 89, 450-466.

77. Burke, D. M., MacKay, D. G., \& James, L. E. Theoretical approaches to language and aging. In T. J. Perfect \& E. A. Maylor (Eds.), Debates in psychology. 
Models of cognitive aging (pp. 204-237). New York, NY, US: Oxford University Press, 2000.

78. Ackerman, P. L., \& Rolfhus, E. L. The locus of adult intelligence: Knowledge, abilities, and non-ability traits. Psychology and Aging, 1999, 14, 314-330.

79. Burke, D. M., MacKay, D. G, Wordslay, J. S. \& Wade, E. 1991. "On the tip of the tonge: What causes wordfinding failures in jounger and older adults". En Journal of memory and language, 1991, 30, 542-579.

80. Baltes, P. B., \& Lindenberger, U. Sensorik und Intelligenz: Inter-systemische Wechselwirkungen und Veranderungen im hohen Alter [Sensory functioning and intelligence: Intersystemic dependencies andchanges in old age]. Akademie-Journal, 1995, 1, 20-28

81. Spar, J., \& La Rue, A. Clinical manual of geriatric psychiatry. 1st Ed. American Psychiatric Publishing, Inc, 2006.

82. Ardila, R. Calidad de vida: una definición integradora. Revista Latinoamericana de Psicología, 2003, 35(2), 161-4.

83. Krzemien, D., Monchietti, A., \& Urquijo, S. Afrontamiento activo y adaptación al envejecimiento en mujeres de la ciudad de Mar del Plata: una revisión de la estrategia de autodistracción, 2005, 22(2), 183-210.

84. Binotti, P., Spina, D., Barrera, M., \& Donolo, D. Funciones ejecutivas y aprendizaje en el envejecimiento normal. Estimulación cognitiva desde una mirada psicopedagógica. Revista Chilena de Neuropsicología, 2009, 4(2), 119-126.

85. Manes, F., \& Torralva, T. Funciones ejecutivas y trastornos del lóbulo frontal. Instituto de Neurología Cognitiva (INECO) Centro de Estudios de la Memoria de Buenos Aires, 2007.

86. Urbano, C., \& Yuni, J. Educación de adultos mayores. Teoría, investigación e intervenciones. Argentina: Brujas, 2005.
87. Frías, L., Guerra, A., Rodríguez, G., \& Guillén, A. Correlación entre el control inhibitorio y la memoria en los adultos mayores. Revista Cubana de Neurologia y Neurocirugía, 2015, 5, 24-29.

88. Gonzalez Aguilar, M. J., Rubio, M., \& Grasso, L. Evaluación del potencial de aprendizaje en adultos mayores: influencia de la mediación en una prueba de memoria. Ciencias Psicológicas, 2018, 12(2), 215-222.

89. Melguizo, E., Acosta, A., \& Castellano, B. Factores asociados a la calidad de vida de adultos mayores. Cartagena 2012, 28 (2), 251-263.

90. Aguilar, K., de los Ángeles, M., \& Herrera, L. Función ejecutiva en adultos mayores con patologías asociadas a la evolución del deterioro cognitivo. Revista Neuropsicología Latinoamericana 2014, 6, 7-14.

91. Ball, K., Berch, D., Helmers, K., Jobe, J., Leveck, M., \& Marsiske, M. (Advanced Cognitive Training for Independent and Vital Elderly Study Group. Effects of cognitive training interventions with older adults. A randomized controlled trial.JAMA, 2002, 288(18), 2271-2281.

92. Montejo, P. Programa de entrenamiento de memoria para mayores con alteraciones de memoria: resultados y predictores. Revista especializada de geriatría y gerontología, 2003, 38(6), 316-326.

93. Valencia, C., López-Alzate, E., Tirado, V., ZeaHerrera, M. D., Lopera, F., Rupprecht, R., \& Oswald, W. D. Efectos cognitivos de un entrenamiento combinado de memoria y psicomotricidad en adultos mayores. Revista de neurología, 2008, 46(8), 465-471.

94. Garamendi, F., Delgado, D., \& Amaya, M. Programa de entrenamiento cognitivo en adultos mayores [Cognitive training program inolder adults]. Revista Mexicana de Medicina Física y Rehabilitación 2010, 22, 26-31. 